\title{
Depolymerization of Poly(Ethylene Terephthalate) Resin Under Pressure
}

\author{
J. Y. CHEN, C. F. OU, Y. C. HU, and C. C. LIN* \\ Institute of Applied Chemistry, National Chiao Tung University, Hsinchu, Taiwan, Republic of China
}

\begin{abstract}
SYNOPSIS
The process of depolymerization of PET resin by EG glycolysis under pressure is investigated. The kinetics of this pressurized depolymerization of PET resin is discussed. It was found that the rate of depolymerization is dependent of temperature, pressure, and concentration ratio of EG to PET. The rate of depolymerization is proportional to the square of EG concentration and faster than that under atmospheric pressure. Glycolyzed products under pressure consist of the PET monomer, BHET, and oligomers, mostly dimer and trimer. An equilibrium between BHE'T and oligomers is attained quickly soon after the depolymerization step is completed in the case of a higher ratio of EG/PET used. In the case of lower ratio of EG / PET, the final product now consists of higher molecular weight of oligomers rather than monomer, dimer, and trimer.
\end{abstract}

\section{INTRODUCTION}

Polyethylene terephthalate (PET) is a semicrystalline thermoplastic used, for example, in the manufacture of high-strength fibers, photographic films, and soft-drink bottles. Because the management of waste plastics is reaching a crisis worldwide, PET bottles have become one of most valuable recyclable materials today. ${ }^{1}$ In addition to mechanical methods of reusing plastics, PET can be reclaimed through chemical means and used to produce new polymer compositions. The various possibilities for utilizing PET product waste have been studied for many years. ${ }^{2-4}$ Most of these studies are related to industrial production of polyols for unsaturated polyester or polyurethane. ${ }^{5-7}$ Recently, Baliga and Wong studied the glycolysis of postconsumer PET drink bottles using ethylene glycol (EG) according to Lin's comment. ${ }^{8}$ Most of former studies on PET glycolysis were, however, carried out under atmospheric pressure. In this study the glycolysis of PET resin under high pressure is presented and its kinetics are dis-

* To whom all correspondence should be addressed. Journal of Applied Polymer Science, Vol. 42, 1501-1507 (1991) (C) 1991 John Wiley \& Sons, Inc. CCC 0021-8995/91/061501-07\$04.00 cussed. In our laboratory we are investigating various possible applications of depolymerization products from PET scraps including from recycled PET bottles.

\section{EXPERIMENTAL}

\section{Materials}

A fiber grade of PET chips with a number average molecular weight range of 18,000-20,000 was obtained from Far Eastern Textile Ltd. A chemical grade of ethylene glycol was purchased from market without further purification.

Table I Hydroxy Value, Degree of Polymerization, and Melting Point

\begin{tabular}{ccc}
\hline$\overline{\mathrm{DP}}(n)$ & $\mathrm{OH}$ Number $(\mathrm{mg} \mathrm{KOH} / \mathrm{g})$ & $\mathrm{mp}\left({ }^{\circ} \mathrm{C}\right)$ \\
\hline 1 & 441.6 & $109 \sim 110$ \\
2 & 251.4 & $168 \sim 170$ \\
3 & 175.6 & $200 \sim 202$ \\
4 & 134.9 & $218 \sim 220$ \\
\hline
\end{tabular}


<smiles>CC(CO)COc1ccc(OCCO)cc1</smiles>

Figure 1 Chemical structure of BHET.

\section{Apparatus}

The PET resin was depolymerized under a pressure interval from 1-6.12 atm and at a temperature interval from $190-240^{\circ} \mathrm{C}$ in a laboratory stirred reactor having a bomb cylinder of $2000 \mathrm{~mL}$ (Parr Instrument) for various reaction times of $0.5,1,2$, and 3 $h$ and at various ratios of EG/PET.

\section{Analysis}

The glycolyzed products and the extracted glycolyzed products were then analyzed for hydroxyl value by the titration method as follows: About $0.5 \mathrm{~g}$ of sample was accurately weighed and added to $10 \mathrm{~mL}$ of acetylating solution, containing $88: 12(\mathrm{v} / \mathrm{v})$ pyridine and acetic anhydride, in a $50-\mathrm{mL}$ flask. The flask was fitted with a vertical reflux condenser and heated with stirring in a boiling water bath for about $2 \mathrm{~h}$. The mixture was then cooled to room temperature and hydrolyzed by $100 \mathrm{~mL}$ of chilled distilled water in another flask. Under vigorous stirring, 20 $\mathrm{mL}$ of benzene were added. The resulting solution was titrated against $0.5 \mathrm{~N} \mathrm{KOH}$ standard using phenolphthalein as indicator. A blank run, i.e., without the sample, was also performed. The hydroxyl value is then calculated as follows:

$$
\mathrm{OH} \text { number }=\frac{\left(V_{\text {blank }}-V_{\text {sample }}\right)[\mathrm{KOH}]}{W_{\text {sample }}}
$$

where the various $V$ s are the volumes of titration, $[\mathrm{KOH}]$ is the molar concentration of titration, and $W$ is the weight of the sample taken. The melting points of the samples were also measured by a capillary dilatometer. Relationships among hydroxyl value, degree of polymerization, and melting point are shown in Table I.

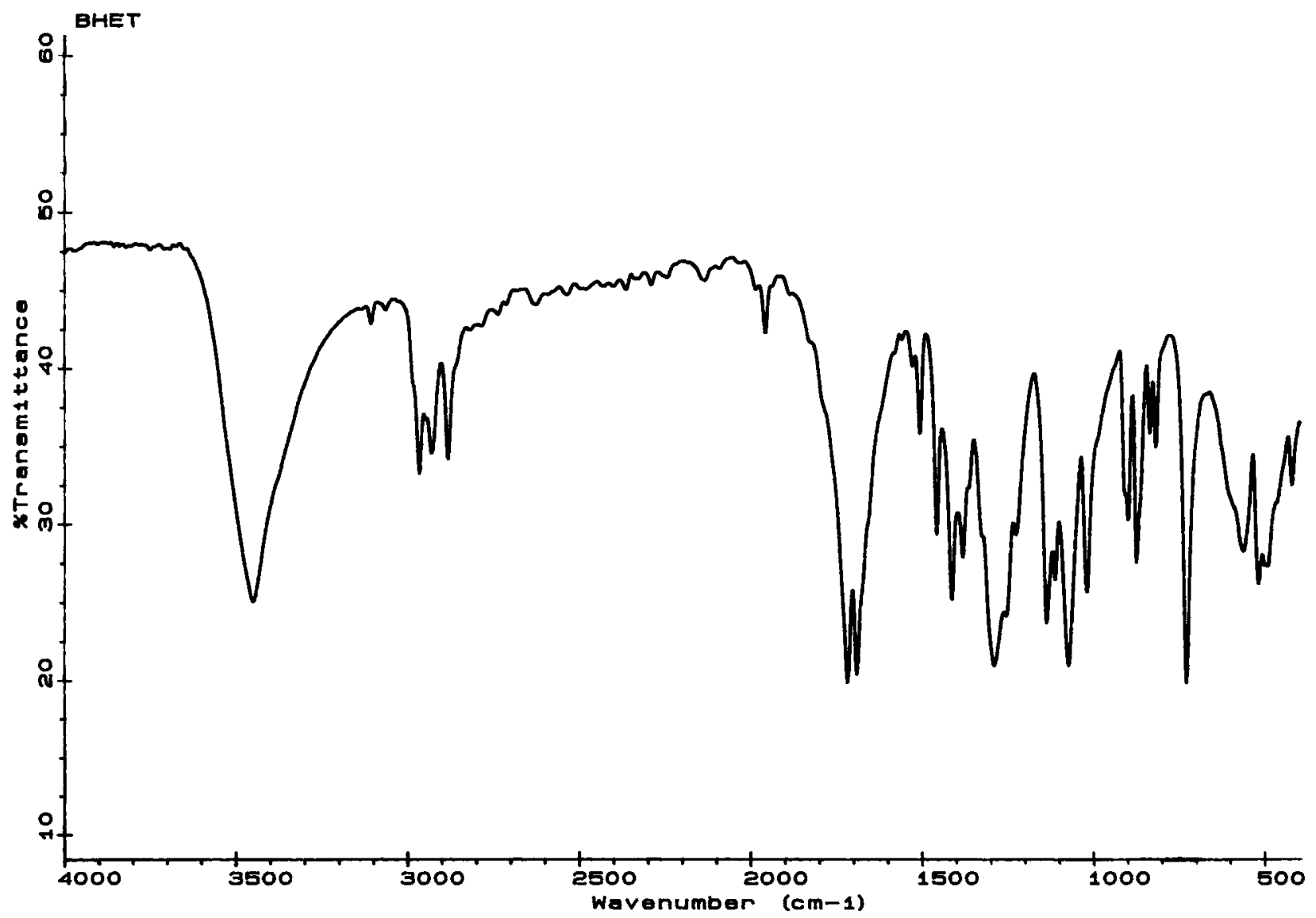

Figure 2 Infrared spectroscopy of BHET. 


\section{RESULTS AND DISCUSSION}

The PET resin can be readily depolymerized by pressurized glycolysis of EG without adding any catalyst. At the end of reaction time, the contents of the reactor were allowed to quench to room temperature under nitrogen atmosphere. The solid products were then stirred vigorously in large amount of water in order to dissolve the unreacted EG. By filtration, the glycolysis products were obtained as residue. This is then labeled as fraction $\mathrm{A}$. The difference between original and final weight represents the amount of free glycol removed by water extraction. A part of fraction A sample was transfered to a beaker and filled with a large amount of distilled water. The suspension was boiled with stirring in order to extract the monomer, e.g., bis(hydroxyethyl terephthalate) (BHET), which has a melting point at $109-110^{\circ} \mathrm{C}$ with the chemical structure as shown in Figure 1.
BHET is known to be quite soluble in boiling water. While still hot, the suspension was quickly filtered. Upon immersing the filtrate in an ice bath, white crystalline flakes were observed to precipitate. These crystals are labeled as fraction $B$, while the residue will be labeled as fraction C. Glycolyzed products of about $80 \mathrm{wt} \%$ are fraction $\mathrm{C}$ for $n=2$ 3 with melting point from $150-180^{\circ} \mathrm{C}$ and about 20 wt $\%$ are fraction $\mathrm{B}$ for $n=1$ with melting point at $110^{\circ} \mathrm{C}$. Figure 2 shows IR spectroscopy of fraction $\mathrm{B}$, indicating a pure BHET structure. The absorptions at $3400,3000-2800,1750$, and $1100 \mathrm{~cm}^{-1}$ are due to $-\mathrm{OH}, \mathrm{C}-\mathrm{H}, \mathrm{C}=\mathrm{O}$, and $\mathrm{C}-\mathrm{O}$ stretchings, respectively. Figure 3 shows NMR spectroscopy of fraction $B$, which identifies the protons in the $A, B$, $\mathrm{C}$, and D chemical surroundings of BHET, respectively. The peaks of DMSO and $\mathrm{H}_{2} \mathrm{O}$ represent solvent and water contaminant.

The hydroxyl number of the glycolyzed products is plotted against glycolysis time. The results are



Figure 3 Nuclear magnetic resonance spectrum of BHET. 


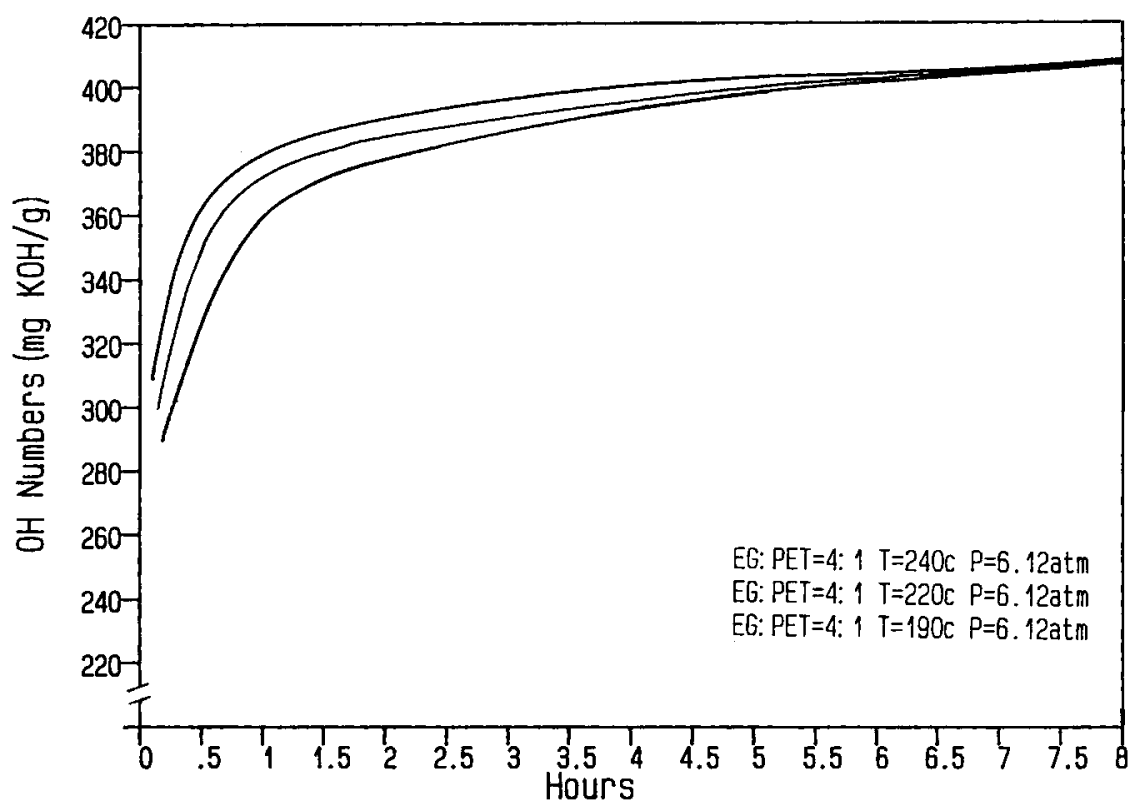

Figure 4 Glycolysis under different temperatures.

shown in Figures 4, 5, and 6, for temperature, pressure, and EG/PET ratio dependences, respectively.

Moreover, the final product depolymerized by using a low EG/PET ratio has a higher molecular weight of oligomer as seen in Figure 6. For instance, the glycolyzed product has a molecular weight around $2000(n=10)$ if $\frac{1}{4}$ of EG/PET ratio is used in the glycolysis. It is clear that a definite amount of EG is needed in order to obtain a given length of oligomers. From these results we believe that the mechanism of pressurized depolymerization of PET resin can be divided into two steps:

$$
\begin{aligned}
\mathrm{PET}+\mathrm{EG} & \rightarrow \text { BHET }+ \text { Oligomers } \\
\mathrm{BHET} & \rightleftharpoons \text { Oligomers }+ \text { EG }
\end{aligned}
$$

The mechanism represented by Eqs. (1) and (2) is different from that proposed by Baliga and Wong. ${ }^{8}$ From our experimental data, the glycolyzed products

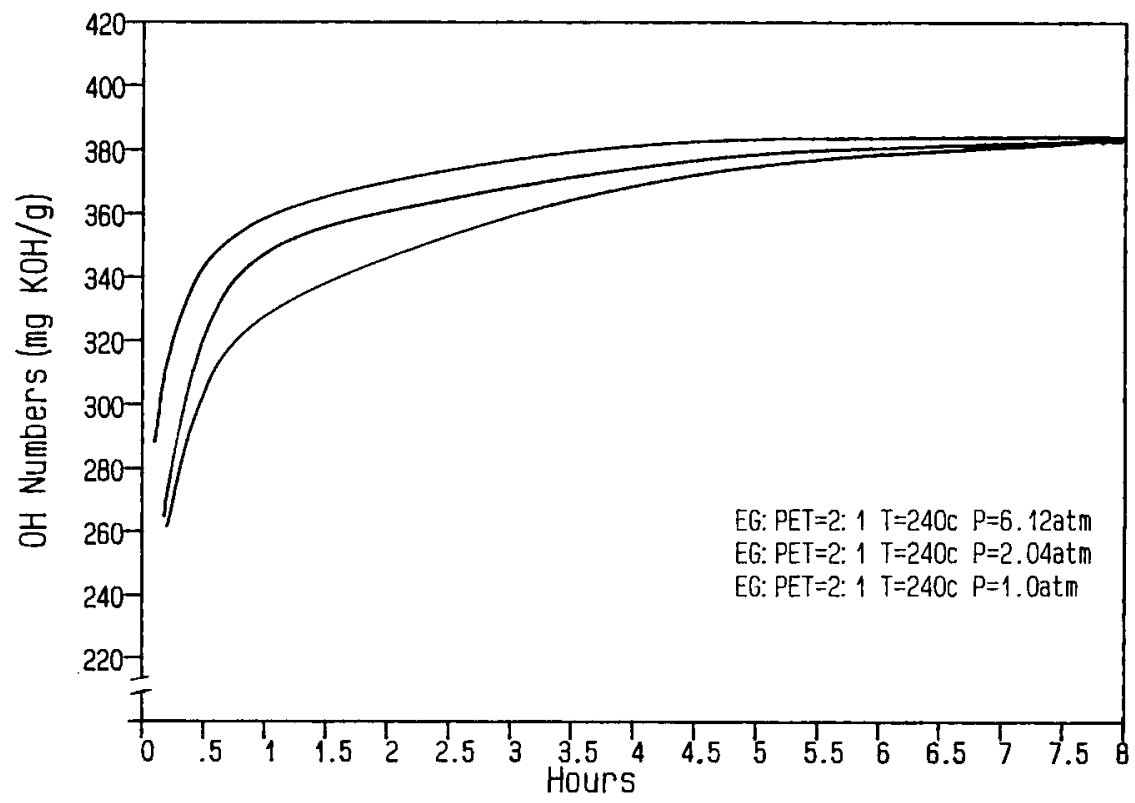

Figure 5 Glycolysis under different pressures. 


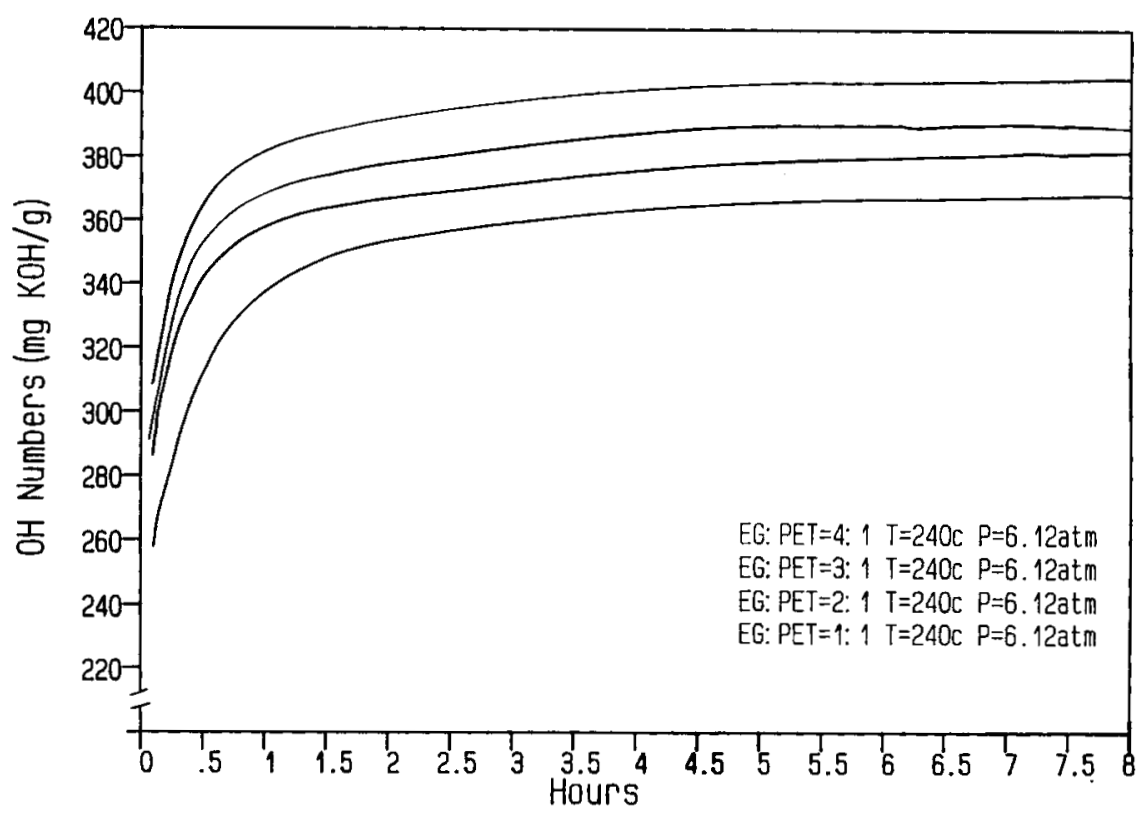

Figure 6 Glycolysis under different EG / PET ratios.

consisted of monomer BHET, dimer, and trimer because fraction $\mathrm{C}$ always consisted of dimer as well as trimer according to the $\mathrm{OH}$ number detected. In fact, one of the reverse-phase liquid chromatogram study by Tong et al. ${ }^{9}$ indicated the existence of dimer as well as trimer in the glycolyzed products. According to our kinetic studies, as shown in Figures $7-9$, the depolymerization rate is dependent on pressure, temperature, and EG/PET ratio, respec- tively, as represented by Eq. (3). The depolymerization rate is expressed here as $\mathrm{OH}$ number/h, which is related to the reciprocal of the degree of polymerization of the samples.

Depolymerization rate $\alpha(P)(T)[\mathrm{EG}] /[\mathrm{PET}]$

where $[E G]$ is defined as the molar concentration

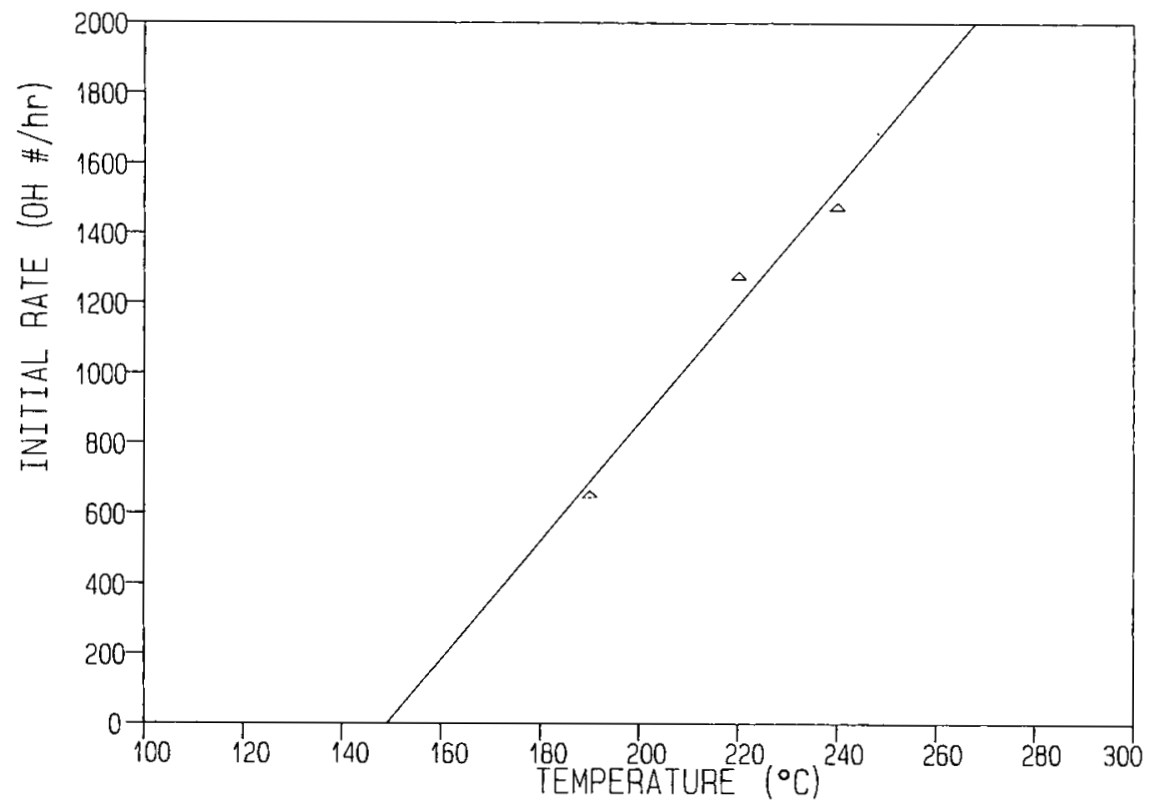

Figure 7 Depolymerization rate vs. temperature. 


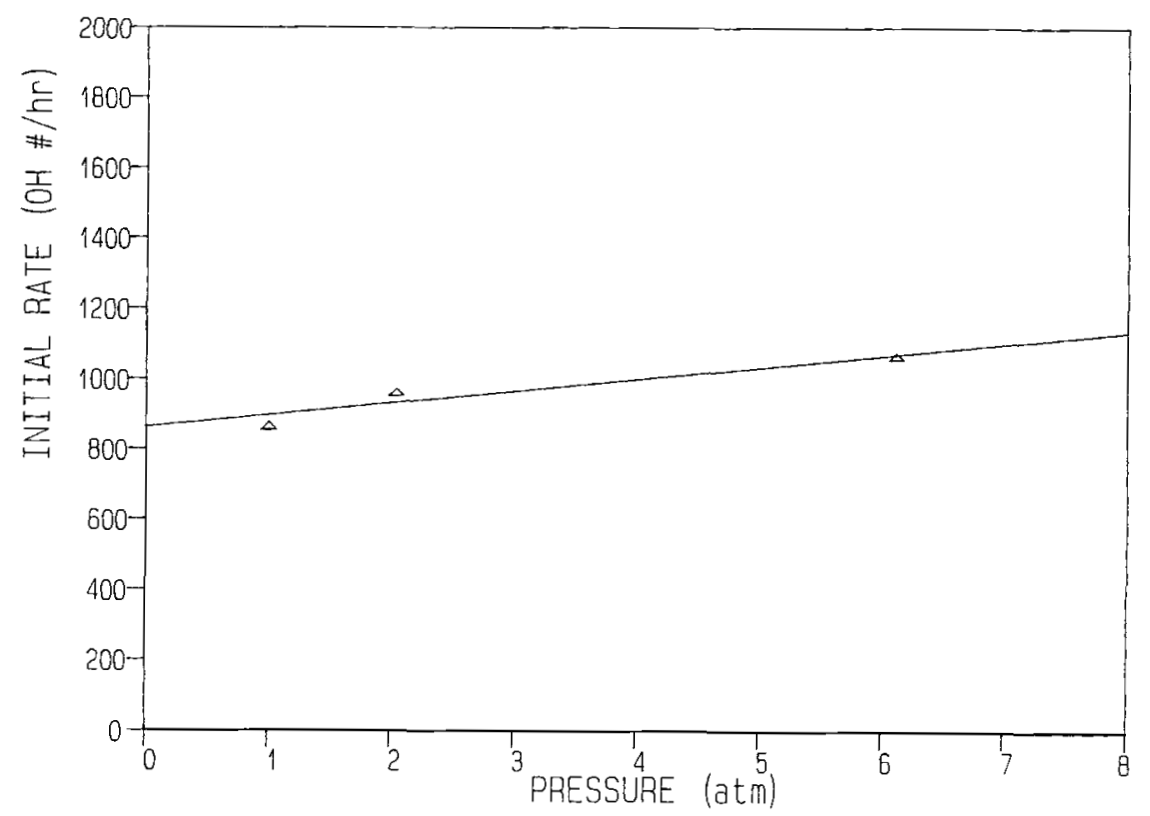

Figure 8 Depolymerization rate vs. pressure.

of EG based on unit mole of PET, and [PET] is defined as the molar concentration of PET based on unit mole of EG. Under the constant pressure, temperature and PET concentration [Eq. (3) ] can be reduced to

$$
\text { Depolymerization rate }=k[\mathrm{EG}]^{2}
$$

A plot of depolymerization rate against the square of EG concentration shows a linear relationship as shown in Figure 9. Based on these kinetics, it is believed that the depolymerization of PET resin is catalyzed by EG. In other words, EG acts as both reactant as well as catalyst in the glycolysis reaction for depolymerization. The reaction step of Eq. (1) is very fast, say the chain scission will be completed within half an hour. After the scission reaction on the polymer chains is completed, there must exist an equilibrium reaction between BHET and oligomers (mostly dimer and trimer) as shown in Eq.

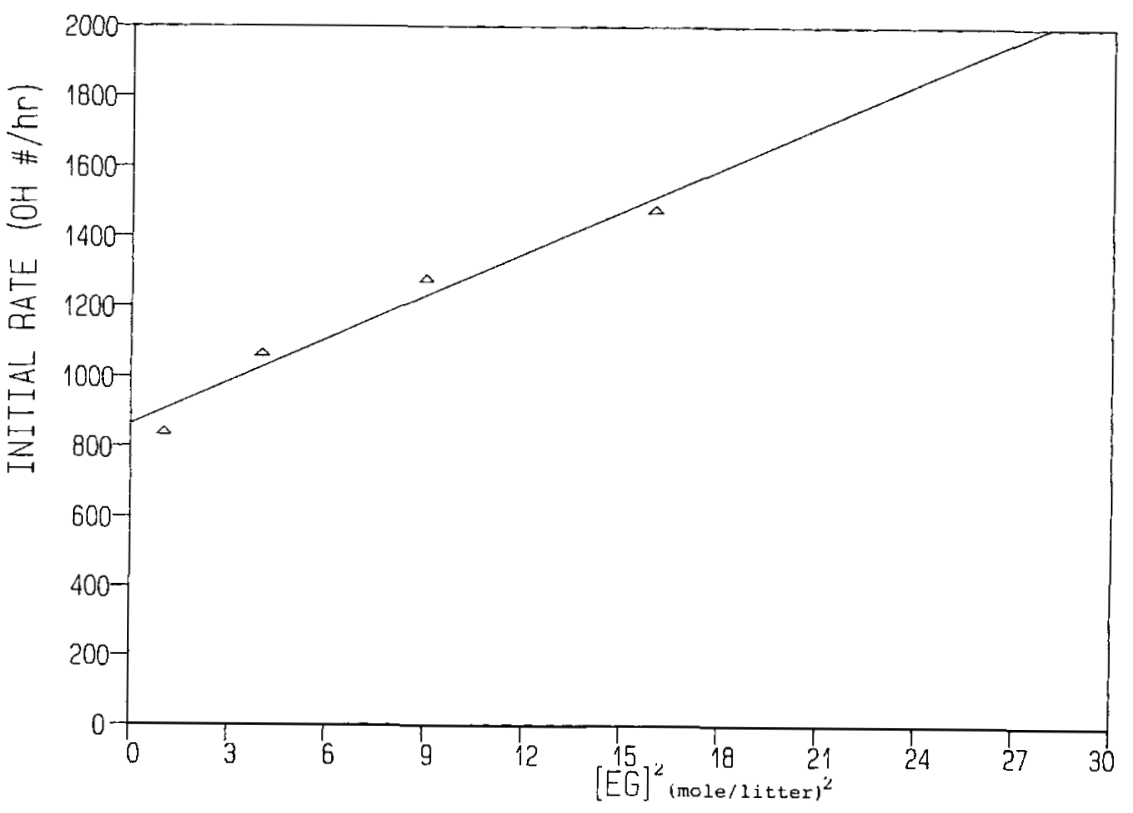

Figure 9 Depolymerization rate vs. $[E G]^{2}$. 
(2). Because the scission reaction happens so quickly and randomly, the oligomers higher than trimer were too minute to be detected in the case of high EG/PET ratio. Howevar, in a low EG/PET ratio, say $\frac{1}{4}$, the glycolyzed product consists of oligomers having a higher molecular weight, say about 2000 of $n=10$. This implies that the degree of polymerization of product may be regulated easily by the EG / PET ratio used in glycolysis. In other words a sufficient amount of EG is needed to depolymerize PET resin to a short-chain length. Equation (4) also indicates that the depolymerization rate is dependent on the square of the molar concentration of EG.

\section{CONCLUSION}

The depolymerization of PET is dependent on pressure, temperature, and EG / PET ratio. The depolymerization rate is very fast and proportional to the square of EG concentration at a constant temperature, pressure, and PET concentration. This implies that EG acts as both reactant and catalyst in the chain scission step. After the scission step of the reaction, there exists an equilibrium between BHET and oligomers that consist mostly of dimer and trimer in the case of high EG / PET ratio. In the case of low EG/PET ratio, the depolymerized products consist of higher molecular weight oligomers. This indicates that a sufficient amount of EG is needed to depolymerize PET resin to obtain a predominant amount of monomer BHET in the glycolyzed products. This also implies that the equilibrium reaction shifts to the rich BHET content side slowly over a very long period of reaction time.

\section{REFERENCES}

1. G. R. Smoluk, Mod. Plast., 65 (2), 87 (1988).

2. A. Fugata, M. Sato, and M. Murakami, U. S. Pat. 4,609,680 (1986).

3. M. Matsuura, T. Habara, and Y. Katagiri, Japan Kobai Pat. 7,57,639 (1975).

4. H. S. Ostrowski, U. S. Pat. $3,884,850$ (1975).

5. U. R. Vaidya and V. M. Nadkarni, Ind. Eng. Chem. Res., 26, 194 (1987).

6. U. R. Vaidya and V. M. Nadkarni, J. Appl. Polym. Sci., 34, 235 (1988).

7. U. R. Vaidya and V. M. Nadkarni, J. Appl. Polym. Sci., 35, 775 ( 1988).

8. S. Baliga and W. T. Wong, J. Polym. Sci., Part A. Polymer Chem., 27, 2071 (1989).

9. S. N. Tong, D. S. Chen, C. C. Chen, and L. Z. Chung, Polymer, 24, 469 (1983).

Received November 16, 1990

Accepted July 9, 1990 